Portland State University

PDXScholar

\title{
Transformation of Pyrococcus furiosus with Thermostable Green Fluorescent Protein
}

\author{
Emma R. Hollar \\ Portland State University
}

Follow this and additional works at: https://pdxscholar.library.pdx.edu/honorstheses

\section{Let us know how access to this document benefits you.}

\section{Recommended Citation}

Hollar, Emma R., "Transformation of Pyrococcus furiosus with Thermostable Green Fluorescent Protein" (2018). University Honors Theses. Paper 609.

https://doi.org/10.15760/honors.620

This Thesis is brought to you for free and open access. It has been accepted for inclusion in University Honors Theses by an authorized administrator of PDXScholar. Please contact us if we can make this document more accessible: pdxscholar@pdx.edu. 
Portland State University

PDXScholar

University Honors Theses

University Honors College

\section{Transformation of Pyrococcus furiosus with Thermostable Green Fluorescent Protein}

Emma R. Hollar

Let us know how access to this document benefits you.

Follow this and additional works at: https://pdxscholar.library.pdx.edu/honorstheses

This Thesis is brought to you for free and open access. It has been accepted for inclusion in University Honors Theses by an authorized administrator of PDXScholar. For more information, please contact pdxscholar@pdx.edu. 


\begin{abstract}
Archaea are the most recently discovered domain of life, are extremely diverse and can be manipulated for research and commercial use. Pyrococcus furiosus is a hyperthermophilic archaeon used as a model organism for studying archaea, especially extremophiles. Green fluorescent proteins are a commonly used marker of transcription but can only be expressed in a narrow temperature zone and denature at the extreme optimal growth temperature of $P$. furiosus. To mediate this inability to use green fluorescent protein in hyperthermophiles, a shuttle vector for $P$. furiosus and Escherichia coli was designed and genes for thermostable green fluorescent proteins were incorporated. This plasmid can be used to upregulate expression of this highly thermostable green fluorescent protein and demonstrate successful transformation. Though the plasmid was not transformed into $P$. furiosus, the construction of the plasmid and transformation of $E$. coli were important steps that gave insight into methods of making this transformation and expression possible.
\end{abstract}

\title{
Introduction
}

Archaea are the most recently discovered and least understood domain of life. Studying their mechanisms for survival and proliferation can tell us a lot about not only our own cells but also how life developed and how to engineer biological systems to exist and flourish in any setting. First described as a distinct domain of life in the 1970s through DNA sequencing, archaea are some of the most diverse and resilient organisms on the planet (Farkas, Picking \& Santangelo 2013). They thrive in conditions with extreme temperatures and salt concentrations, environments previously considered to be uninhabitable. They have also been found in the 
human biome and have important broad environmental impacts such as nitrogen fixation. Archaea were initially classified as bacteria but through sequencing of their genomes, they were discovered as a unique domain of life that serves as an intermediate between bacteria and eukaryotes.

This new classification is important as it distinguishes the mechanisms of archaea as unique, though related to bacteria and eukaryotes. Because of their relatively recent discovery, they are not well studied. There is an immense amount of diversity between species and a wide range of metabolic ability and function. There are also distinct similarities in their molecular mechanisms to bacterial and eukaryotic systems. Studying processes of replication, transcription, and translation in archaea can provide information about the evolution of these essential functions and how they are adapted to extreme conditions.

Archaea have been utilized in microbiological, molecular and medical research to create new compounds, therapies, and treatments. Because archaea have the capacity to survive in extreme conditions and survive in various environments, they have a variety of mechanisms for creating the specific compounds that allow this survival. These systems can be engineered to be used commercially, for research and medicine. The CRISPR/Cas9 system for genomic engineering is a prime example of how microbial systems can be manipulated to benefit humans. The repeated palindromic sequences that are fundamental to this microbial immune system were found in $90 \%$ of archaea (Mojica, Díez-Villaseñor, Soria, \& Juez 2000). The 
significant amount of diversity in the organisms that host this system indicate the potential for a wide variety of useful sequences (Hsu, Lander, \& Zhang 2014).

This project focuses on transcription and transformation in the hyperthermophile Pyrococcus furiosus. This species is a well-studied archaeal model organism, and transforming $P$. furiosus can unravel some of the mysteries involving transcription in hyperthermophiles. The goal of this project is to construct a plasmid that contains a gene for expression of a thermostable green fluorescent protein and transform it into $P$. furiosus.

\section{Background}

Pyrococcus furiosus is a marine hyperthermophilic archaeon originally found in geothermal marine sediments (Fiala \& Stetter 1986). The name Pyrococcus furiosus essentially means "fast-moving fireball". This genus was first isolated off the beach of Porto di Levante, Vulcano Island, Italy. They are obligate anaerobes and have an optimum growth temperature of $100^{\circ} \mathrm{C}$. The organism is spherical with a multi-tailed flagellum. The organism uses its flagella for both motility and adhesion (Näther, Rachel, Wanner \& Wirth 2006). The flagella form intercellular connections and biofilms which allow them to attach securely to solid surfaces. $P$. furiosus is heterotrophic and utilizes pyruvate as its carbon source (Schäfer \& Schönheit 1991). P. furiosus is used as a model organism to study archaea because it can be easily manipulated and survives in extreme conditions (Lipscomb et al. 2011). The strain used in this experiment, COM1, is a genetically engineered mutant with the pyrF gene knocked out of the genome. This strain can 
be used for further manipulation by integrating plasmids that contain the pyrF gene as well as other reporter genes and selectable markers.

Green fluorescent proteins (GFP) are a common marker of transformation in bacterial and eukaryotic systems but are ineffective in hyperthermophilic archaea because of their temperature sensitivity (Henche, Koerdt, Ghosh \& Albers 2012). GFPs will not fold properly in the high temperatures that some archaea require for optimal growth and therefore cannot be used as an indicator of transformation. A thermostable GFP gene, eCGP123 was designed and originally used in thermophilic bacteria (Kiss, Temirov, Chasteen, Waldo \& Bradbury 2009). This gene allowed expression of the fluorescent protein as a marker for transformation in organisms that survive at higher temperatures.

The protein was constructed from a previous form of fluorescent protein by consensus engineering, which modifies and aligns the target protein's sequence to resemble similar sequences. A recursive evolutionary process was then used to stabilize the protein through mutation and select for the mutants that were able to function after this evolution. eCGP was first successfully transformed into the hyperthermophilic archaeon, Sulfolobus acidocaldarius and used as a measure of biofilm growth (Henche, Koerdt, Ghosh \& Albers 2012). In this project, eCGP will also be used as a reporter gene but we will also use a different gene, TGP in the hope that this will give a higher yield of fluorescent protein. 
The target reporter gene for this project, TGP, was derived from eCGP123. Though eCGP was a heat-stable, successful alternative to traditional green fluorescent protein, it was found to aggregate easily and fall out of solution (Close et al. 2015). Structure-guided surface engineering was used to modify amino acid residues on the outside of the protein. These changes to the protein altered the surface charge to make the protein more negative for increased stability and destabilization of interactions between the amino acids in adjacent proteins that leads to aggregation. TGP has not been previously used as a marker for transformation in archaea.

The plasmid chosen for transformation is pYS4 (Figure 1). This plasmid was designed as a shuttle vector specifically for P. furiosus (Waege, Schmid, Thumann, Thomm \& Hausner 2010). The vector has since been modified by Charles Wilson in the Bartlett lab to contain a selectable marker, pyrF and in this study has been engineered to contain copies of either TGP or eCGP. By using vectors such as pYS4, the organisms can be modified to be resistant to different antibiotics, express novel proteins and metabolize new compounds. Adapting the pYS4 shuttle vector system in $P$. furiosus allows the creation of a set of tools that can be used to modify archaea in a way that can be useful for commercial and research purposes. 


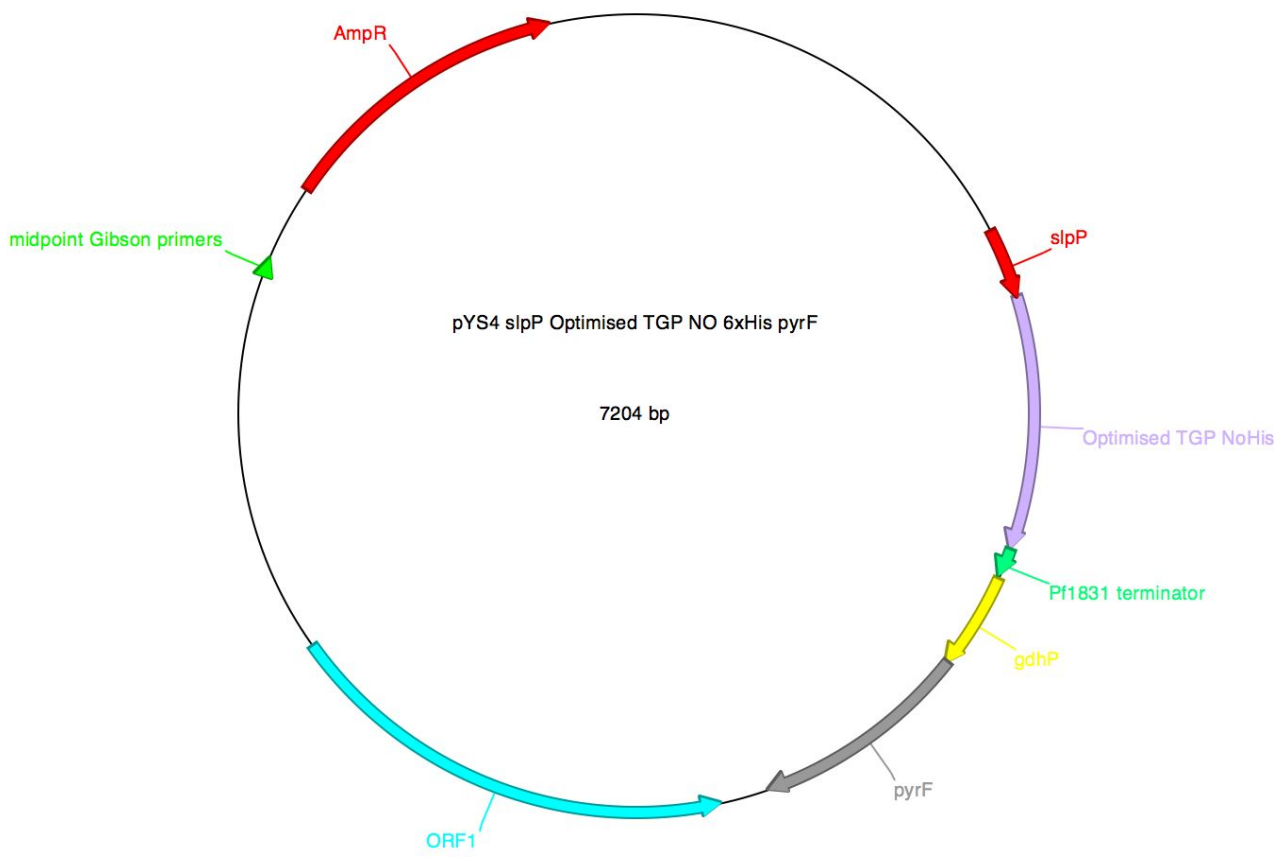

Figure 1: pYS4 plasmid with TGP based on sequencing data. The initial plasmid was constructed by Charles Wilson (D(2)3) and was used as a template to amplify backbone fragments pyrF (pyrF to midpoint) and slpP (promoter to midpoint). TGP was the third fragment in the assembly (lavender). The plasmid containing the eCGP gene features the same construction but with the TGP replaced with eCGP. Both fragments are approximately the same size.

\section{Methods}

The aim of this project was to transform the archaeon $P$. furiosus with plasmid pYS4 containing the TGP reporter gene for expression of the thermostable green fluorescent protein. To assemble the plasmid, gene fragments were amplified, purified and quantified. The three fragments isolated to construct the plasmid were the pyrF selectable marker, slpP promoter and the target gene (eCGP123 or TGP). Gibson Assembly was used to create the plasmid. The Gibson Assembly reactions were then transformed into competent Escherichia coli to select for viable transformants. The plasmids were then sequenced. Following verification of assembly, 
they will then be transformed into $P$. furiosus. The reporter gene will indicate whether GFP is functional in this system through measurement of fluorescence.

The first step of the project involves amplifying DNA fragments for assembly. This was done using polymerase chain reaction (PCR). The four fragments were pYS4 pyrF, pYS4 slpP, TGP and eCGP. The pyrF and slpP fragments were amplified from a pYS4 plasmid previously assembled by Charles Wilson in the Bartlett Lab which was confirmed by sequencing in January 2018. The TGP and eCGP fragments were isolated and confirmed by PCR in July 2016. Each reaction was performed using 5x HF buffer and a thermostable DNA polymerase, Phusion by Fermentas.

The template was Charles Wilson's D(2)3 pYS4 for the pYS4 pyrF fragment and the primers were pYS4 NOHisStop and MidToSel. The template for the pYS4 slpP fragment was also $D(2) 3$ and the primers were pYS4 MidToExp and sIpP rev universal. The template for TGP amplification wass the previously purified TGP from July 2016 and the primers were TGP for slpoverlap and pYS4 TGP NoHisRev. The template for eCGP was the previously purified eCGP from July 2016 and the primers were eCGP for slpoverlap and pYS4 eCGP NoHisRev.

Once confirmed by PCR, the fragments were purified using a Fermentas plasmid purification kit and quantified. Gibson Assembly was used to construct the plasmid. The three Gibson Assembly reactions were an slpP only control, pyrF + slpP + TGP and pyrF +slpP + eCGP. 15 fmol of backbone fragment (pyrF) and $50 \mathrm{fmol}$ of each slpP fragment and reporter gene were used 
for each reaction. The reactions were transformed into competent $E$. coli XL1 Blue and plated on LB medium with $100 \mu \mathrm{g} / \mathrm{ml}$ carbenicillin and grown at $37^{\circ} \mathrm{C}$ for 24 hours.

Isolated colonies were selected and streaked for colony PCR. Colonies were selected by examination under $365 \mathrm{~nm}$ UV light. Colonies that appeared to fluoresce were streaked onto a second LB carb100 plate. Multiple isolates from each reaction were screened using the forward primer that corresponded to the reporter gene and the reverse primer, gdhL, to examine the junctions between the slpP and the reporter gene as well as the gdhP promoter and the reporter gene to ensure that the reporter gene had been incorporated. The sequences were then confirmed by the OHSU sequencing lab by D-mode sequencing. For TGP and eCGP pYS4 constructions, each plasmid was sequenced through multiple reactions, using forward and reverse primers for each junction.

The resulting sequences were compared with previous sequences by looking at the alignment of each fragment against the expected sequences. The plasmids with confirmed sequences will then be transformed into $P$. furiosus strain COM1 to select for viable transformants. These can be identified using the selectable marker pyrF in the plasmid which will allow growth on media lacking uracil. Viable transformants will be checked for fluorescence under UV light due to the expression of TGP or eCGP in the plasmid. 


\section{Results}

We have not yet successfully transformed the plasmid into $P$. furiosus COM1. Each fragment was amplified (Figure 2) after multiple unsuccessful attempts by optimizing the quantities of reagents in the PCR mixture. The pyrF fragment was the most difficult to amplify and generally resulted in very small quantities for each PCR. Multiple reactions were carried out and combined to have a high enough concentration of pyrF for Gibson Assembly. The assembly was transformed into competent $\mathrm{E}$. coli and selected by carbenicillin resistance as well as and fluorescence under 365 nm UV light (Figure 3).

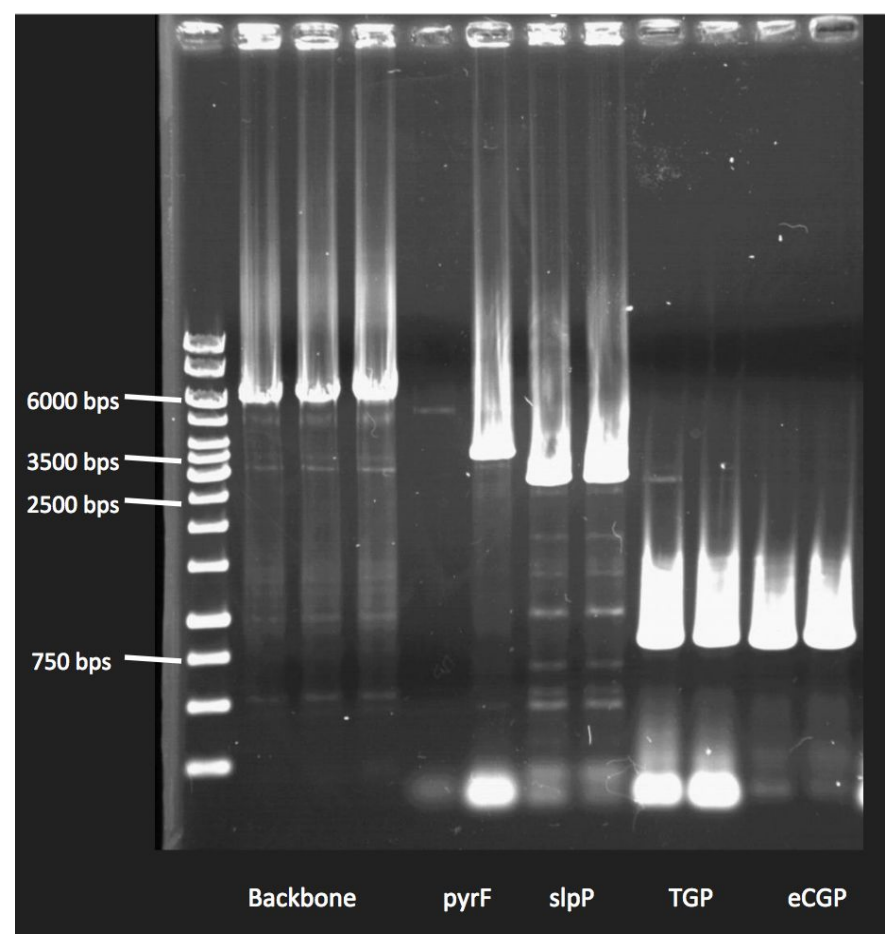

Figure 2: fragments used to construct the pYS4 plasmid and each reporter gene, TGP and eCGP. Expected sizes of each fragment were 6466 base pairs for the pYS4 backbone, 3643 base pairs for the pyrF fragment, 2648 base pairs for the slpP fragment, 738 base pairs for TGP and 734 base pairs for eCGP. 

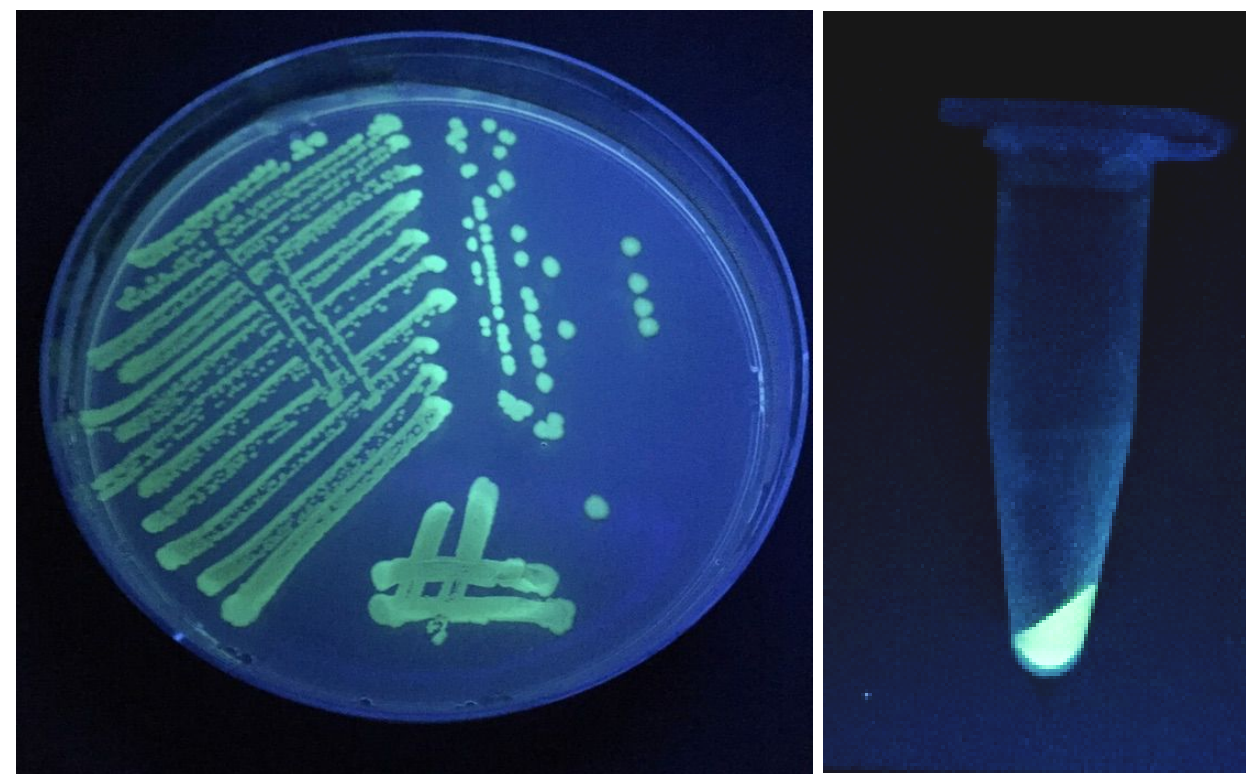

Figure 3: $E$. coli expressing the transformed plasmid containing the TGP gene. This expression is an intermediate step intended to first express the plasmid in E.coli and screen for viable tranformants. The plasmids are then purified from the cells, sequenced and transformed into P. furiosus.

The plasmid was purified and prepared for sequencing, but the sequence data indicated that the plasmid had not assembled correctly and that only the slpP and reporter gene fragments were present. The misassembly was confirmed by a gel that showed plasmids missing approximately 3600 base pairs, which was the size of the pyrF fragment (Figure 4). 


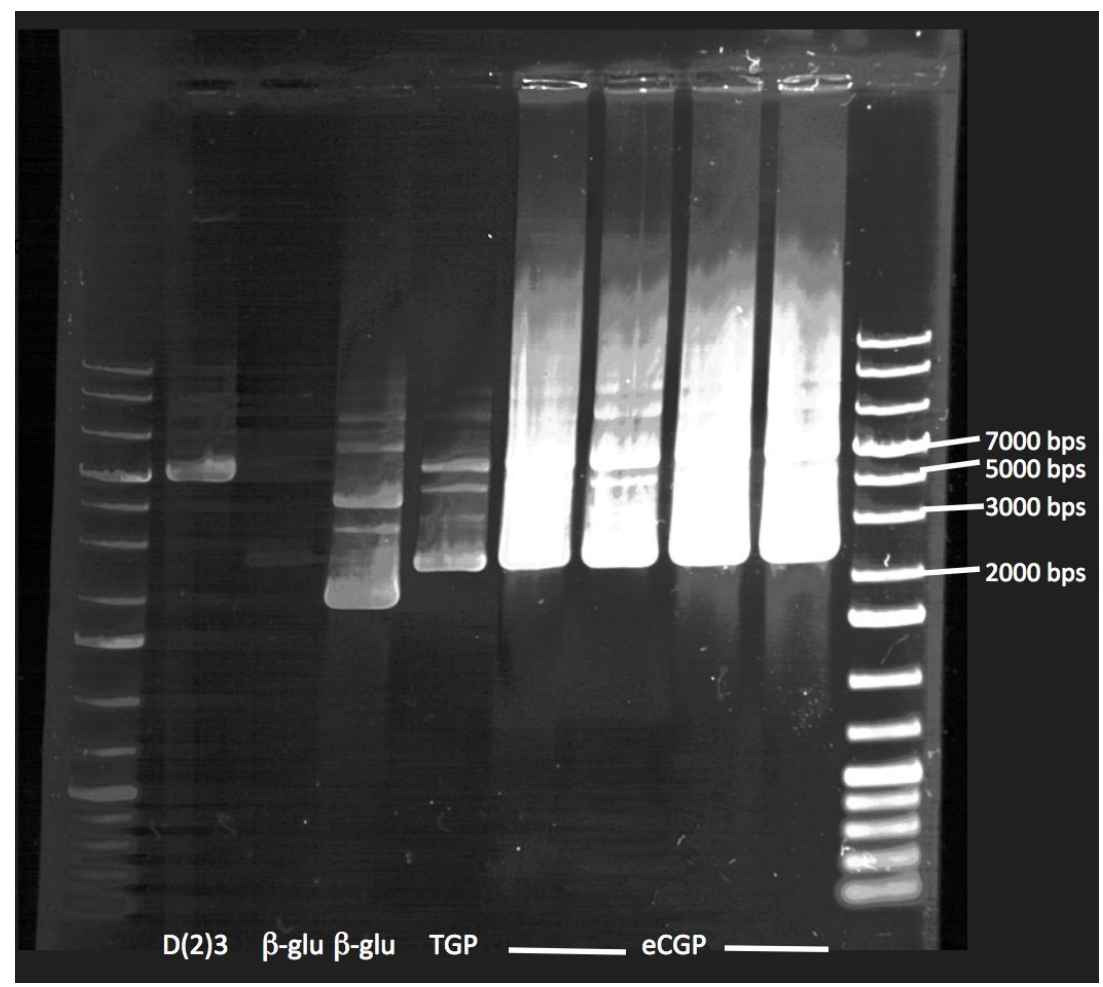

Figure 4: screen of plasmids isolated by colony PCR from fluorescing $E$. coli colonies with positive control pYS4 plasmid D(2)3. This gel demonstrates the size discrepancy between the $D$ (2)3 plasmid confirmed by sequencing and the isolated plasmids from transformed $\mathrm{E}$. coli. The size of D(2)3 and expected size of each plasmid was approximately 7200 base pairs but the resulting plasmids were only approximately 2500 base pairs, confirming the missing pyrF fragment as evidenced by the sequencing results.

The plasmid was reassembled using the initial fragment amplifications and re-transformed and purified but the colony PCR screen gave the same results as the first assembly and sequencing reactions came back inconclusive. The fragments were re-amplified using $D(2) 3$ as a template for the pyrF and slpP fragments as well as amplification across the entire plasmid backbone (Figures 1, 2 \& 3). The thermocycler's gradient feature was utilized to optimize the annealing temperature for the Phusion polymerase. In the previously used PCR program designed for the Phusion, there was a touchdown for annealing at $56^{\circ} \mathrm{C}, 54^{\circ} \mathrm{C}, 52^{\circ} \mathrm{C}$ and $50^{\circ} \mathrm{C}$. A diagnostic screening was done to determine the optimal annealing temperature with six samples of each 
fragment at a temperature range of $48^{\circ} \mathrm{C}$ to $60^{\circ} \mathrm{C}$. Twelve reactions with the backbone fragment were conducted simultaneously at each temperature interval. The optimal temperature was found to be $54^{\circ} \mathrm{C}$. The remaining fragment amplifications with Phusion utilized this annealing temperature.

The plasmid was reassembled by Gibson Assembly using $15 \mathrm{fmol}$ of backbone and $50 \mathrm{fmol}$ of TGP or eCGP. The same procedure for transformation and selection was used and the colonies were screened for fluorescence. Selected colonies' plasmids were screened with the reporter gene's forward primer and gdhR reverse primer to ensure the gene had been properly incorporated. Sequencing has verified correct assembly of the plasmid.

\section{Discussion}

The reality of a novel construction, such as the integration of a new gene and plasmid into a strain, is that many variables contribute to success and the process may require a significant amount of trial and error. The transformation of $P$. furiosus with the engineered plasmid will be possible, but the engineering process required more adjustments than the time frame of this project allowed. As each step of the experiment was carried out, the successes and failures indicated what worked and what needed to be adjusted. The three main issues faced in this project were the differences between $P$. furiosus and E. coli, the amplification and assembly of the fragments and low transformation rates once the plasmid was assembled. 
Transformation of Pyrococcus furiosus with Thermostable Green Fluorescent Protein

Though the final goal of the experiment was to transform the plasmid into P. furiosus, an intermediate step involved transformation into E. coli to purify and isolate properly constructed plasmids. This step ensures that the plasmid has assembled correctly and can be sequenced before transformation into COM1. P. furiosus and E. coli, are very different organisms with varied mechanisms and will potentially have different responses to the plasmid. $E$. coli is a bacterium that grows best aerobically and has an optimum growth temperature of $37^{\circ} \mathrm{C}$. P. furiosus is an obligate anaerobe and grows at $100^{\circ} \mathrm{C}$. Though the plasmid is specifically designed for transformation in both $P$. furiosus and $E$. coli, integration and expression are possible .

The plasmid was first designed as a shuttle vector between $P$. furiosus and $E$. coli, giving transformants resistance to the antibiotic simvastatin (Waege et al. 2010). The plasmid was redesigned multiple times to first optimize it for $P$. furiosus and and remove HMG CoA reductase (conferring simvastatin resistance) in exchange for the pyrF gene. After multiple experiments, this decision was made to substitute the selectable marker because simvastatin was unreliable, degraded easily at high temperature and a substantial concentration could not be determined. Ampicillin resistance is commonly used in $E$. coli and has already defined effective concentrations. Carbenicillin is a close relative of ampicillin and is a useful and more stable alternative, and was effective for E. coli transformation at a standard concentration of $100 \mu \mathrm{g} / \mathrm{ml}$.

The initial construction of the plasmid involved amplifying three different fragments, two from the backbone and one reporter gene, TGP or eCGP. These fragments were based on midpoint 
Gibson primers determined by the sequence of the plasmid (Figure 1). The initial amplifications of the fragments from the $D(2) 3$ plasmid were unsuccessful. The amplification was optimized by increasing the amount of template DNA used in each reaction mixture. 10x HF Phusion polymerase buffer was also determined to have the best results. The best template for pyrF and slpP was the plasmid, as using previous amplifications as templates led to extra fragment formation that may have affected assembly. A similar result was observed with TGP and eCGP so the stock GBlock TGP and eCGP were used as a template for reporter gene amplification.

Once the concentrations of each fragment were quantified, the plasmid was assembled by Gibson Assembly. The protocol called for $15 \mathrm{fmol}$ of backbone, which was the pyrF fragment in this construction, and $50 \mathrm{fmol}$ of the remaining fragments which were slpP and TGP or eCGP. The higher concentration of additional fragments is used in an attempt to create a discrepancy between the backbone and target fragments and force them into the plasmid. This attempts to ensure that the backbone will not close and anneal on itself and instead incorporate the higher amounts of the desired fragment. The concentrations of the fragment are limited by quantity as the reaction protocol only allows for a total of $2.5 \mu$ of purified PCR product. The concentrations of each fragment must be high enough that the required amount of DNA can be included in a very small volume.

The purified pyrF fragment was only at a concentration of $11 \mathrm{fmol} / \mu \mathrm{l}$ which was less than the required $15 \mathrm{fmol}$. Because the slpP and TGP fragments were also to be included in the assembly, only $1 \mu$ l of pyrF was used in the first Gibson Assembly. This lower concentration than 
Transformation of Pyrococcus furiosus with Thermostable Green Fluorescent Protein

the protocol called for was a potential reason of why there was improper assembly for the first round of $E$. coli transformation. The pyrF fragment was known to be missing because the colonies were able to grow on carbenicillin plates and fluoresce, indicating that the slpP fragment with the ampicillin resistance gene and the TGP or eCGP fragments coding for expression of the fluorescent protein, had been integrated. This was confirmed by a screen of the plasmids that showed their size to be only approximately 3600 base pairs instead of the expected size of 7200 base pairs. The pyrF fragment is 3643 base pairs in length which would account for the missing half of the plasmid.

"To address the problem of misassembly, multiple amplifications of pyrF were performed and combined, and the plasmid was reassembled with a higher pyrF concentration but gave the same results. Because this assembly appeared to be ineffective, the entire backbone was amplified and combined in a Gibson Assembly reaction with the TGP or eCGP fragments and transformed into competent $E$. coli. These transformants were screened with UV for fluorescence and the gene was examined with the forward primer that corresponded (TGP or eCGP for slpoverlap) and reverse primer gdhR. This ensured that the gene incorporated into the right place in the backbone. By using only the backbone instead of the slpP and pyrF fragments, the chance of improper assembly is lowered.

Though there was successful transformation of the plasmid into $E$. coli, there was a low amount of colonies that appeared to fluoresce on each transformation plate. Out of approximately 250 colonies produced for each transformation, less than 10 had evidently acquired the TGP or 
eCGP genes. This could be due to the fact that the strength of fluorescence seemed to increase with time or temperature. An observation was made that the colonies appeared more green to the naked eye after spending 24 hours in the fridge which would indicate the cells required more time to express the protein or that the protein took time to mature and become fluorescent. It is unlikely that the lower temperature upregulated expression due to its stability at extremely high temperatures but it could be a possible variable for investigation. The low transformation rate could also be attributed to the fact that pYS4 is designed for $P$. furiosus instead of $E$. coli and there could be a discrepancy in the organisms' expression mechanisms.

The most likely cause of low transformation rates is that pYS4 is a high copy number plasmid. The origin of replication used in the plasmid construction comes from a commonly used cloning for transformation, pUC19 (Waege, Schmid, Thumann, Thomm \& Hausner 2010). pUC19 is a high copy plasmid and is 2686 base pairs in length (New England Biolabs 2018). It easy easily engineered and often used as a vector because it has multiple restriction endonuclease cleavage sites. Its application as a high copy plasmid works well due to its relatively small size. When the pUC19 origin of replication is used in the construction of another plasmid, the high copy properties are transferred along with it. This means that any expression of the new plasmid will also be in high quantities. This property is not conducive for the transformation of large plasmids such as pYS4. The length of pYS4 with TGP and eCGP is 7204 base pairs which is a much greater size for the cells to express. "The large size and high quantity of this plasmid may overload the cellular machinery, and affect viability of cells containing the plasmid. 
The high copy origin of replication is a possible explanation for the low transformation rates observed in E. coli. The cells most likely aren't able to handle the large amount of replication directed by the plasmid. The plasmid could also be toxic to the cells, possibly due to the high quantity of DNA they are forced to produce and express. This could also be an issue when transformed into $P$. furiosus. To mediate this, the plasmid will be redesigned to have a low copy number origin. This should ensure that the machinery will not be overloaded and should be able to express properly. Using a standard origin of replication should result in higher levels of transformation. The brightness of fluorescence between the high copy origin plasmid and the reworked standard origin plasmid should be compared to see if there is a variation in expression when there are fewer copies of the vector.

Other observations made throughout the experiment were the appearance of some of the $E$. coli colonies after transformation. A small amount of the colonies were smaller, clear and glassy looking in contrast to the sticky, white robust appearance of wild-type $E$. coli. The clear colonies did not contain properly assembled plasmid and did not express the fluorescent proteins. When these were selected and streaked onto a fresh LB carb100 plate, they would not grow as well as normal or properly transformed clones. The vector was likely toxic to these cells possibly due to improper assembly or mutation and prevented them from replicating. 
Transformation of Pyrococcus furiosus with Thermostable Green Fluorescent Protein

\section{Conclusion}

Though the project was not completed before the end of Spring 2018, many experiments providing significant and important information were conducted. The difficulty in amplifying the fragments for Gibson Assembly led to the optimization of PCR and experimentation with the concentrations required for the assembly protocol. The sequencing data gave extremely significant results by indicating the plasmid had not assembled correctly even though the reporter genes were being expressed. The low transformation rates and use of high copy origin of replication from pUC19 for a large plasmid together suggest that the high quantity of replication the cells must undergo is possibly toxic and prevents growth.

The next steps in keeping the aim of this experiment viable involve re-engineering the plasmid to have a standard origin of replication. This should lower the amount of replication and DNA burden on the cells and allow them to grow and transform better. For further synthesis and assembly, the entire plasmid backbone should be amplified all at once instead of in multiple fragments. The new version of pYS4 combined with the lessons learned in terms of Gibson Assembly should result in higher transformation rates but may have an effect on expression. Each step of the experiment revealed new problems that must be reworked. Each new observation made or protocol modified adds up, until the desired result is achieved.

This project ultimately offers a new mechanism for ensuring transformation in a model organism. As archaea are being more intimately studied and understood, there is becoming an increased need for techniques to engineer and manipulate the organism. There is a wide 
diversity in archaea, but there is a significant lack of knowledge and tools for practical research and commercial use. The application of this widely used fluorescent protein to hyperthermophilic organisms would greatly expand the opportunities that this unique organism provides us. By optimizing a vector and reporter gene system for Pyrococcus furiosus, there can be nearly endless possibilities of what the cells can be manipulated to do, produce and survive.

\section{Acknowledgements}

This project could not have been possible or enjoyable without the work done by others in the Bartlett Lab. Thank you to Dr. Bartlett for being the most patient and enjoyable thesis advisor, Charles Wilson for assembly of the pYS4 plasmid used and Arman Carrasco for being an absolute joy to work every step of the project. Also thanks to Farshid Taghizadeh, Kimberly Sheffield, Leakna Sok and Ermina Kariuki for support and simultaneous experiments that contributed to this one. I also appreciate the help, insight and support from my parents, siblings, friends and roommate who all contributed in their unique, individual ways. 


\section{Bibliography}

Close, D. W., Paul, C. D., Langan, P. S., Wilce, M. C., Traore, D. A., Halfmann, R., ... \& Prescott, M. (2015). Thermal green protein, an extremely stable, nonaggregating fluorescent protein created by structure-guided surface engineering. Proteins: Structure, Function, and Bioinformatics, 83(7), 1225-1237.

Farkas, J. A., Picking, J. W., \& Santangelo, T. J. (2013). Genetic techniques for the archaea. Annual review of genetics, 47, 539-561.

Fiala, G., \& Stetter, K. O. (1986). Pyrococcus furiosus sp. nov. represents a novel genus of marine heterotrophic archaebacteria growing optimally at $100 \mathrm{C}$. Archives of Microbiology, 145(1), 56-61.

Henche, A. L., Koerdt, A., Ghosh, A., \& Albers, S. V. (2012). Influence of cell surface structures on crenarchaeal biofilm formation using a thermostable green fluorescent protein.

Environmental microbiology, 14(3), 779-793.

Hsu, P. D., Lander, E. S., \& Zhang, F. (2014). Development and applications of CRISPR-Cas9 for genome engineering. Cell, 157(6), 1262-1278.

Kiss, C., Temirov, J., Chasteen, L., Waldo, G. S., \& Bradbury, A. R. (2009). Directed evolution of an extremely stable fluorescent protein. Protein Engineering, Design \& Selection, 22(5), 313-323.

Lipscomb, G. L., Stirrett, K., Schut, G. J., Yang, F., Jenney, F. E., Scott, R. A., ... \& Westpheling, J. (2011). Natural competence in the hyperthermophilic archaeon Pyrococcus furiosus facilitates genetic manipulation: construction of markerless deletions of genes encoding the two cytoplasmic hydrogenases. Applied and environmental microbiology, 77(7), 2232-2238.

Mojica, F. J., Díez-Villaseñor, C., Soria, E., \& Juez, G. (2000). Biological significance of a family of regularly spaced repeats in the genomes of Archaea, Bacteria and mitochondria. Molecular microbiology, 36(1), 244-246.

Näther, D. J., Rachel, R., Wanner, G., \& Wirth, R. (2006). Flagella of Pyrococcus furiosus: multifunctional organelles, made for swimming, adhesion to various surfaces, and cell-cell contacts. Journal of Bacteriology, 188(19), 6915-6923. 
New England Biolabs. (2018). PUC19 Vector.

Schäfer, T., \& Schönheit, P. (1991). Pyruvate metabolism of the hyperthermophilic archaebacterium Pyrococcus furiosus. Archives of microbiology, 155(4), 366-377.

Waege, I., Schmid, G., Thumann, S., Thomm, M., \& Hausner, W. (2010). Shuttle vector-based transformation system for Pyrococcus furiosus. Applied and environmental microbiology, 76(10), 3308-3313. 